\title{
Bio efficacy of Newer Insecticide Molecules against Safflower Capsule Borer, Helicoverpa armigera (Hubner)
}

\author{
K.A. Biradarpatil ${ }^{1 *}$ and S.B. Jagginavar ${ }^{2}$ \\ ${ }^{1}$ Agricultural Entomology, University of Agricultural Sciences, Dharwad, Karnataka, India \\ ${ }^{2}$ Agricultural Entomology, College of Agriculture, Vijayapur, University of Agricultural \\ Sciences, Dharwad, Karnataka, India \\ *Corresponding author
}

\section{A B S T R A C T}

\section{Keywords \\ Bio efficacy, Insecticide molecules, Helicoverpa armigera}

Article Info

Accepted: 04 June 2018 Available Online: 10 July 2018

\begin{abstract}
A field experiment was conducted during rabi season of 2013-14 at Agricultural College Farm, Vijayapur by using a safflower variety Annigeri-1. The results indicated that, among different insecticides tested against capsule borer, the lowest capsule borer per plant was recorded in rynaxypyr $20 \mathrm{SC} @ 0.15 \mathrm{ml} / \mathrm{l}$ and was on par with emamectin benzoate 5\% SG @0.20 gm/l, novaluron10 EC @ 0.75ml/1. The highest yield and benefit cost ratio was recorded in rynaxypyr 20 SC @ 0.15 $\mathrm{ml} / 1$ followed by emamectin benzoate 5\% SG @ 0.20 gm/1 and spinosad 45 SC @ $0.1 \mathrm{ml} / \mathrm{l}$. The maximum number of predators Viz., C. zastrowi and coccinellids was noticed in Biodigester@1:3 ratios, NSKE 5\%, NPV@250 LE/ha, Btk@1 $\mathrm{gm} / \mathrm{ha}$.
\end{abstract}

\section{Introduction}

Safflower (Carthamus tinctorius Linn.) is an important rabi oilseed crop in semi arid areas of India. Apart from India, it is cultivated in Mexico, Australia, Mediterranean Europe, California valley, China and Africa (Anon, 1995). In India, safflower production remained stagnant at about 554 thousand tones. In India, Maharashtra is the highest producer of safflower (63\%) from the largest growing area $(67 \%)$ followed by Karnataka in production $32 \%$ and in area $27 \%$ (Jadhav et al., 2012).
Among the several factors responsible for low production of safflower, insect pests have been considered as one of the important biotic factors. A total of 101 pests are known to attack the safflower at different stages of crop growth (Patil and Halolli, 2005). The important insect pest includes capsule fly (Acanthiophilus helianthi Rossi), capsule borer (Helicoverpa armigera Hub.) and safflower aphid (Uroleucon carthami L.) (Karve et al., 1978). The damage caused by capsule borer ( $H$. armigera) surpass the loss caused by all insect pests together by their direct damage to the economically important 
parts (capsule and leaf) of plant and it has been claimed that the loss due to this pest range from 62.6 to 100 per cent (Sekhar and Rai, 1989).

The liberal use of many chemical insecticides led to problems like toxic residues, elimination of natural enemies, environmental disharmony and development of resistance. Hence, the present study was carried out to evaluate the efficacy of some newer insecticides against $H$. armigera in safflower along with available conventional insecticides.

\section{Materials and Methods}

A field trial was conducted during rabi season of 2013-14 at Agricultural College Farm, vijayapur by using a safflower variety Annigeri-1. Randomized block design was followed with 14 treatments and 3 replications. The crop was sown at a spacing $60 \times 30 \mathrm{~cm}$ over a plot size of $5 \times 4.8 \mathrm{sq}$. m by following all the agronomic practices. Application of botanicals, bioagents and chemical insecticides was taken up by using high volume knapsack sprayer at the rate of 500 liters of spray fluid per hectare. The incidence of $H$. armigera was recorded as number of larvae per plant at one day before treatment while post-treatment counts were taken at 3, 10 and 15 days after spraying of insecticides. The grain yield was recorded from each plot separately and converted to per hectare. The data on incidence of $H$. armigera and yields were subjected to suitable statistical analysis. The mean values of the treatments were statistically analyzed and then separated by Duncan`s Multiple Range Test (DMRT).

\section{Results and Discussion}

The results revealed that during rabi 2013-14, All the insecticidal treatments, including organic products were significantly effective than untreated control in reducing the larva of capsule borer population (Table 1). 3 days after first spray (DAS) lowest incidence of $H$. armigera larva was recorded with rynaxypyr 20 SC @ 0.15ml/1 (0.18/plant) and it was significantly superior over all other treatments. The next best treatments were novaluron 10 EC @ 0.75ml/1, emamectin benzoate 5 SG @ $0.2 \mathrm{~g} / \mathrm{l}$, spinosad 45 SC @ $0.10 \mathrm{ml} / 1$ and flubendiamide 480 SC @ 0.075ml/1 recorded 0.19 larvae per plant when compared to 1.10 larvae per plant in control plot (Table 1). Same trend was observed during 10 DAT and 15 DAT.

This finding is in agreement with the results of Satpute and Barkhade (2012) who reported that rynaxypyr $20 \mathrm{SC}$ was found effective in reducing larval population in pigeon pea. Deshmukh et al., (2010) opined that insecticidal treatments with flubendiamide @ 0.007 per cent gave significantly highest mortality of the pest in chickpea. Patil et al., (2007) suggested that emamectinbenzoate was very effective in reducing the larval population in chickpea. Rajesh et al., (2010) recorded that rynaxypyr $20 \mathrm{SC} @ 30 \mathrm{~g}$ a.i /ha and rynaxypyr 20 SC @ 20 g. a.i/ha were superior in recording the less larval population of fruit borer in okra.

All treatments proved profitable over control and increase in yield varied from the maximum of $10.68 \mathrm{q} \mathrm{ha}^{-1}$ in rynaxypyr to the minimum of $7.65 \mathrm{q} \mathrm{ha}{ }^{-1}$ in biodigester treatment (Table 2). The gross income incurred due to the different treatments was the highest (Rs. 34176) in case of rynaxypyr while it was the lowest (Rs 24480) in biodigester. Remaining treatments occupied intermediate position yet with wide difference in respect of gross income. The net profit derived out of different treatments got affected since the cost of production in the these treatments ranged from a minimum of Rs. $10620 \mathrm{ha}^{-1}$ in case of acephate to the maximum of Rs. $11452 \mathrm{ha}^{-1}$ in case of rynaxypyr. 
Table.1 Bioefficacy of different insecticides against capsule borer in safflower

\begin{tabular}{|c|c|c|c|c|c|c|}
\hline \multirow[t]{2}{*}{$\begin{array}{l}\text { Tr. } \\
\text { No. }\end{array}$} & \multirow[t]{2}{*}{ Treatment } & & \multicolumn{4}{|c|}{$\begin{array}{c}\text { Mean larval population of capsule borer } \\
\text { per plant }\end{array}$} \\
\hline & & Dosage & 1DBT & 3 DAT & $10 \mathrm{DAT}$ & $15 \mathrm{DAT}$ \\
\hline $\mathbf{T}_{1}$ & Profenophos 50 EC & $2 \mathrm{ml} / 1$ & $\begin{array}{c}0.83 \\
(1.15)\end{array}$ & $\begin{array}{c}0.22 \\
(0.85)^{\mathrm{abc}}\end{array}$ & $\begin{array}{c}0.23 \\
(0.85)^{\mathrm{ab}}\end{array}$ & $\begin{array}{l}0.23 \\
(0.85)^{\mathrm{abc}}\end{array}$ \\
\hline $\mathbf{T}_{2}$ & Acephate 75 SP & $1 \mathrm{gm} / \mathrm{l}$ & $\begin{array}{c}0.97 \\
(1.21)\end{array}$ & $\begin{array}{c}0.23 \\
(0.85)^{\mathrm{abc}}\end{array}$ & $\begin{array}{c}0.24 \\
(0.86)^{\mathrm{abc}}\end{array}$ & $\begin{array}{l}0.26 \\
(0.87)^{\mathrm{abc}}\end{array}$ \\
\hline $\mathbf{T}_{3}$ & NSKE $5 \%$ & $5 \mathrm{ml} / 1$ & $\begin{array}{c}0.91 \\
(1.19)\end{array}$ & $\begin{array}{c}0.43 \\
(0.96)^{\mathrm{c}}\end{array}$ & $\begin{array}{c}0.44 \\
(0.97)^{\mathrm{c}}\end{array}$ & $\begin{array}{c}0.47 \\
(0.98)^{\mathrm{bc}}\end{array}$ \\
\hline $\mathbf{T}_{4}$ & Deltamethrin 10EC & $0.5 \mathrm{ml} / 1$ & $\begin{array}{c}0.83 \\
(1.15)\end{array}$ & $\begin{array}{c}0.24 \\
(0.86)^{\mathrm{abc}}\end{array}$ & $\begin{array}{l}0.25 \\
(0.87)^{\mathrm{abc}}\end{array}$ & $\begin{array}{c}0.27 \\
(0.88)^{\mathrm{abc}}\end{array}$ \\
\hline $\mathbf{T}_{5}$ & NPV $2.0 \%$ AS & $250 \mathrm{LE} / \mathrm{ha}$ & $\begin{array}{c}0.79 \\
(1.14)\end{array}$ & $\begin{array}{c}0.39 \\
(0.94)^{\mathrm{bc}}\end{array}$ & $\begin{array}{c}0.41 \\
(0.95)^{\mathrm{bc}}\end{array}$ & $\begin{array}{l}0.44 \\
(0.97)^{\mathrm{bc}}\end{array}$ \\
\hline $\mathbf{T}_{6}$ & Btk $0.5 \% \mathrm{WP}$ & $1 \mathrm{gm} / 1$ & $\begin{array}{c}0.82 \\
(1.15)\end{array}$ & $\begin{array}{c}0.41 \\
(0.95)^{\mathrm{bc}}\end{array}$ & $\begin{array}{l}0.44 \\
(0.97)^{\mathrm{c}}\end{array}$ & $\begin{array}{l}0.46 \\
(0.98)^{\mathrm{bc}}\end{array}$ \\
\hline $\mathbf{T}_{7}$ & Novaluron $10 \mathrm{EC}$ & $0.75 \mathrm{ml} / \mathrm{l}$ & $\begin{array}{c}0.82 \\
(1.15)\end{array}$ & $\begin{array}{c}0.19 \\
(0.83)^{\mathrm{ab}}\end{array}$ & $\begin{array}{l}0.19 \\
(0.83)^{\mathrm{a}}\end{array}$ & $\begin{array}{c}0.21 \\
(0.84)^{\mathrm{ab}}\end{array}$ \\
\hline $\mathbf{T}_{8}$ & Rynaxypyr 20 SC & $0.15 \mathrm{ml} / \mathrm{l}$ & $\begin{array}{c}0.75 \\
(1.12)\end{array}$ & $\begin{array}{c}0.18 \\
(0.82)^{\mathrm{a}}\end{array}$ & $\begin{array}{c}0.18 \\
(0.82)^{\mathrm{a}}\end{array}$ & $\begin{array}{c}0.19 \\
(0.83)^{\mathrm{a}}\end{array}$ \\
\hline $\mathbf{T}_{9}$ & Spinosad 45 SC & $0.1 \mathrm{ml} / 1$ & $\begin{array}{c}0.83 \\
(1.15)\end{array}$ & $\begin{array}{c}0.19 \\
(0.83)^{\mathrm{ab}}\end{array}$ & $\begin{array}{c}0.2 \\
(0.84)_{\mathrm{ab}}\end{array}$ & $\begin{array}{c}0.2 \\
(0.84)^{\mathrm{ab}}\end{array}$ \\
\hline $\mathbf{T}_{10}$ & Indoxacarb $14.5 \mathrm{SC}$ & $0.3 \mathrm{ml} / 1$ & $\begin{array}{c}0.95 \\
(1.20)\end{array}$ & $\begin{array}{c}0.22 \\
(0.85)^{\mathrm{abc}}\end{array}$ & $\begin{array}{c}0.23 \\
(0.85)^{\mathrm{ab}}\end{array}$ & $\begin{array}{c}0.25 \\
(0.87)^{\mathrm{abc}}\end{array}$ \\
\hline $\mathbf{T}_{11}$ & $\begin{array}{l}\text { Emamectin benzoate } \\
5 \mathrm{SG}\end{array}$ & $0.2 \mathrm{~g} / \mathrm{l}$ & $\begin{array}{c}0.90 \\
(1.18)\end{array}$ & $\begin{array}{c}0.19 \\
(0.82)^{\mathrm{ab}}\end{array}$ & $\begin{array}{c}0.19 \\
(0.83)^{\mathrm{a}}\end{array}$ & $\begin{array}{c}0.21 \\
(0.84)^{\mathrm{ab}}\end{array}$ \\
\hline $\mathbf{T}_{12}$ & Flubendiamide $480 \mathrm{SC}$ & $0.075 \mathrm{ml} / 1$ & $\begin{array}{c}0.89 \\
(1.18)\end{array}$ & $\begin{array}{c}0.19 \\
(0.83)^{\mathrm{ab}}\end{array}$ & $\begin{array}{c}0.2 \\
(0.84)^{\mathrm{ab}}\end{array}$ & $\begin{array}{c}0.22 \\
(0.85)^{\mathrm{abc}}\end{array}$ \\
\hline $\mathbf{T}_{13}$ & Control & --- & $\begin{array}{c}0.91 \\
(1.19)\end{array}$ & $\begin{array}{c}1.1 \\
(1.26)^{\mathrm{e}}\end{array}$ & $\begin{array}{c}1.24 \\
(1.35)^{\mathrm{e}}\end{array}$ & $\begin{array}{c}1.35 \\
(1.36)^{\mathrm{e}}\end{array}$ \\
\hline $\mathbf{T}_{14}$ & Biodigester & $\begin{array}{l}1: 3 \\
\text { (Biodigester } \\
\text { :water) }\end{array}$ & $\begin{array}{c}0.94 \\
(1.20)\end{array}$ & $\begin{array}{c}0.72 \\
(1.10)^{\mathrm{d}}\end{array}$ & $\begin{array}{c}0.84 \\
(1.16)^{\mathrm{d}}\end{array}$ & $\begin{array}{c}0.89 \\
(1.18)^{\mathrm{d}}\end{array}$ \\
\hline & S.Em \pm & & & 0.042 & 0.040 & 0.045 \\
\hline & CD @5\% & & NS & 0.12 & 0.11 & 0.13 \\
\hline & $\mathrm{CV}(\%)$ & & & 7.97 & 7.35 & 8.11 \\
\hline
\end{tabular}

NS - Non-significant, Means followed by same letter in a column do not differ significant by DMRT at 5\% level DBT-Days Before Treatment, DAT - Days After Treatment 
Table.2 Economics of insecticidal treatments on safflower capsule borer

\begin{tabular}{|c|c|c|c|c|c|c|c|}
\hline $\begin{array}{l}\text { Tr. } \\
\text { No. }\end{array}$ & Treatment & $\begin{array}{l}\text { Yield } \\
\text { (q/ha) }\end{array}$ & $\begin{array}{c}\text { Gross } \\
\text { income } \\
\text { (Rs./ha) }\end{array}$ & $\begin{array}{c}\text { Net } \\
\text { income } \\
\text { (Rs./ha) }\end{array}$ & $\begin{array}{c}\text { Cost of } \\
\text { protection } \\
\text { (Rs./ha) }\end{array}$ & $\begin{array}{c}\text { Cost of } \\
\text { production } \\
\text { (Rs./ha) }\end{array}$ & $\begin{array}{l}\mathrm{BC} \\
\text { ratio }\end{array}$ \\
\hline$T_{1}$ & Profenophos 50 EC & $9.83^{\mathrm{ab}}$ & 31456 & 20416 & 740 & 11040 & $1: 1.85$ \\
\hline $\mathbf{T}_{2}$ & Acephate 75 SP & $9.56^{\mathrm{ab}}$ & 30592 & 19972 & 320 & 10620 & $1: 1.88$ \\
\hline $\mathbf{T}_{3}$ & NSKE 5\% & $7.76^{\mathrm{cd}}$ & 24832 & 14032 & 500 & 10800 & $1: 1.30$ \\
\hline $\mathbf{T}_{4}$ & Deltamethrin 10EC & $9.68^{\mathrm{ab}}$ & 30976 & 20246 & 430 & 10730 & $1: 1.89$ \\
\hline $\mathbf{T}_{5}$ & NPV $2.0 \%$ AS & $7.92^{\mathrm{cd}}$ & 25344 & 14494 & 550 & 10850 & $1: 1.34$ \\
\hline $\mathbf{T}_{6}$ & Btk $0.5 \% \mathrm{WP}$ & $7.70^{\mathrm{cd}}$ & 24640 & 13830 & 510 & 10810 & $1: 1.28$ \\
\hline $\mathbf{T}_{7}$ & Novaluron $10 \mathrm{EC}$ & $10.43^{\mathrm{a}}$ & 33376 & 21472 & 1604 & 11904 & $1: 1.80$ \\
\hline $\mathbf{T}_{8}$ & Rynaxypyr 20 SC & $10.68^{\mathrm{a}}$ & 34176 & 22724 & 1152 & 11452 & $1: 1.98$ \\
\hline $\mathbf{T}_{\mathbf{9}}$ & Spinosad 45 SC & $10.47^{\mathrm{a}}$ & 33504 & 22054 & 1150 & 11450 & $1: 1.93$ \\
\hline $\mathbf{T}_{10}$ & Indoxacarb $14.5 \mathrm{SC}$ & $8.96^{\mathrm{bc}}$ & 28672 & 17472 & 900 & 11200 & $1: 1.56$ \\
\hline$T_{11}$ & $\begin{array}{l}\text { Emamectin benzoate } \\
5 \mathrm{SG}\end{array}$ & $10.38^{\mathrm{a}}$ & 33216 & 22086 & 830 & 11130 & $1: 1.96$ \\
\hline $\mathbf{T}_{12}$ & Flubendiamide $480 \mathrm{SC}$ & $10.11^{\mathrm{ab}}$ & 32352 & 21247 & 805 & 11105 & $1: 1.91$ \\
\hline $\mathbf{T}_{13}$ & Control & $6.12^{\mathrm{e}}$ & 19584 & 9284 & 0 & 10300 & 1:0.90 \\
\hline $\mathbf{T}_{14}$ & Biodigester & $7.65^{\mathrm{d}}$ & 24480 & 13580 & 600 & 10900 & $1: 1.25$ \\
\hline & S.Em \pm & 0.46 & & & & & \\
\hline & CD @5\% & 1.35 & & & & & \\
\hline & $\mathrm{CV}(\%)$ & 8.83 & & & & & \\
\hline
\end{tabular}

Means followed by same letter in a column do not differ significant by DMRT at 5\% level, Market price of safflower: 3200/q, cost of cultivation: 10300 
Table.3 Population of coccinelids in chemical sprayed plot in safflower

\begin{tabular}{|c|c|c|c|c|c|c|}
\hline \multirow[t]{2}{*}{$\begin{array}{l}\text { Tr. } \\
\text { No. }\end{array}$} & \multirow[t]{2}{*}{ Treatment } & & \multicolumn{4}{|c|}{$\begin{array}{l}\text { Mean larval population of coccinelid } \\
\text { per plant }\end{array}$} \\
\hline & & Dosage & DBT & $3 \mathrm{DAT}$ & $10 \mathrm{DAT}$ & $15 \mathrm{DAT}$ \\
\hline $\mathbf{T}_{1}$ & Profenophos 50 EC & $2 \mathrm{ml} / 1$ & $\begin{array}{c}0.51 \\
(1.00)\end{array}$ & $\begin{array}{c}0.33 \\
(0.91)^{\mathrm{b}}\end{array}$ & $\begin{array}{c}0.33 \\
(0.91)^{\mathrm{b}}\end{array}$ & $\begin{array}{c}0.31 \\
(0.90)^{\mathrm{c}}\end{array}$ \\
\hline $\mathbf{T}_{2}$ & Acephate 75 SP & $1 \mathrm{gm} / 1$ & $\begin{array}{c}0.48 \\
(0.99)\end{array}$ & $\begin{array}{c}0.35 \\
(0.92)^{\mathrm{b}}\end{array}$ & $\begin{array}{c}0.34 \\
(0.92)^{\mathrm{b}}\end{array}$ & $\begin{array}{c}0.32 \\
(0.91)^{\mathrm{bc}}\end{array}$ \\
\hline $\mathbf{T}_{\mathbf{3}}$ & NSKE 5\% & $5 \mathrm{ml} / \mathrm{l}$ & $\begin{array}{c}0.56 \\
(1.03)\end{array}$ & $\begin{array}{c}0.48 \\
(0.99)^{\mathrm{ab}}\end{array}$ & $\begin{array}{c}0.48 \\
(0.99)^{\mathrm{ab}}\end{array}$ & $\begin{array}{c}0.47 \\
(0.98)^{\mathrm{bc}}\end{array}$ \\
\hline $\mathbf{T}_{4}$ & Deltamethrin 10EC & $0.5 \mathrm{ml} / 1$ & $\begin{array}{c}0.43 \\
(0.96)\end{array}$ & $\begin{array}{c}0.37 \\
(0.93)^{\mathrm{b}}\end{array}$ & $\begin{array}{c}0.36 \\
(0.93)^{\mathrm{b}}\end{array}$ & $\begin{array}{c}0.35 \\
(0.92)^{\mathrm{bc}}\end{array}$ \\
\hline $\mathbf{T}_{5}$ & NPV $2.0 \%$ AS & $250 \mathrm{LE} / \mathrm{ha}$ & $\begin{array}{c}0.49 \\
(0.99)\end{array}$ & $\begin{array}{c}0.43 \\
(0.96)^{\mathrm{ab}}\end{array}$ & $\begin{array}{c}0.43 \\
(0.96)^{\mathrm{ab}}\end{array}$ & $\begin{array}{c}0.41 \\
(0.95)^{\mathrm{bc}}\end{array}$ \\
\hline $\mathbf{T}_{6}$ & Btk $0.5 \% \mathrm{WP}$ & $1 \mathrm{gm} / 1$ & $\begin{array}{c}0.47 \\
(0.98)\end{array}$ & $\begin{array}{c}0.42 \\
(0.96)^{\mathrm{ab}}\end{array}$ & $\begin{array}{c}0.41 \\
(0.95)^{\mathrm{ab}}\end{array}$ & $\begin{array}{c}0.41 \\
(0.95)^{\mathrm{bc}}\end{array}$ \\
\hline $\mathbf{T}_{7}$ & Novaluron $10 \mathrm{EC}$ & $0.75 \mathrm{ml} / \mathrm{l}$ & $\begin{array}{c}0.53 \\
(1.01)\end{array}$ & $\begin{array}{c}0.32 \\
(0.91)^{\mathrm{b}}\end{array}$ & $\begin{array}{c}0.30 \\
(0.89)^{\mathrm{b}}\end{array}$ & $\begin{array}{c}0.30 \\
(0.89)^{\mathrm{c}}\end{array}$ \\
\hline $\mathbf{T}_{8}$ & Rynaxypyr $20 \mathrm{SC}$ & $0.15 \mathrm{ml} / \mathrm{l}$ & $\begin{array}{c}0.59 \\
(1.04)\end{array}$ & $\begin{array}{c}0.35 \\
(0.92)^{\mathrm{b}}\end{array}$ & $\begin{array}{c}0.33 \\
(0.91)^{\mathrm{b}}\end{array}$ & $\begin{array}{c}0.31 \\
(0.90)^{\mathrm{c}}\end{array}$ \\
\hline $\mathbf{T}_{9}$ & Spinosad $45 \mathrm{SC}$ & $0.1 \mathrm{ml} / \mathrm{l}$ & $\begin{array}{c}0.56 \\
(1.03)\end{array}$ & $\begin{array}{c}0.32 \\
(0.91)^{\mathrm{b}}\end{array}$ & $\begin{array}{c}0.31 \\
(0.90)^{\mathrm{b}}\end{array}$ & $\begin{array}{c}0.31 \\
(0.90)^{\mathrm{c}}\end{array}$ \\
\hline $\mathbf{T}_{10}$ & Indoxacarb $14.5 \mathrm{SC}$ & $0.3 \mathrm{ml} / 1$ & $\begin{array}{c}0.51 \\
(1.00)\end{array}$ & $\begin{array}{c}0.36 \\
(0.93)^{\mathrm{b}}\end{array}$ & $\begin{array}{c}0.34 \\
(0.92)^{\mathrm{b}}\end{array}$ & $\begin{array}{c}0.33 \\
(0.91)^{\mathrm{bc}}\end{array}$ \\
\hline $\mathbf{T}_{11}$ & $\begin{array}{l}\text { Emamectin benzoate } \\
\text { 5SG }\end{array}$ & $0.2 \mathrm{~g} / \mathrm{l}$ & $\begin{array}{c}0.48 \\
(0.99)\end{array}$ & $\begin{array}{c}0.33 \\
(0.91)^{\mathrm{b}}\end{array}$ & $\begin{array}{c}0.32 \\
(0.91)^{\mathrm{b}}\end{array}$ & $\begin{array}{c}0.31 \\
(0.90)^{\mathrm{c}}\end{array}$ \\
\hline $\mathbf{T}_{12}$ & Flubendiamide $480 \mathrm{SC}$ & $0.075 \mathrm{ml} / \mathrm{l}$ & $\begin{array}{c}0.53 \\
(1.01)\end{array}$ & $\begin{array}{c}0.32 \\
(0.91)^{b}\end{array}$ & $\begin{array}{c}0.32 \\
(0.91)^{\mathrm{b}}\end{array}$ & $\begin{array}{c}0.31 \\
(0.90)^{\mathrm{c}}\end{array}$ \\
\hline $\mathbf{T}_{13}$ & Control & --- & $\begin{array}{c}0.46 \\
(0.98)\end{array}$ & $\begin{array}{c}0.58 \\
(1.04)^{\mathrm{a}}\end{array}$ & $\begin{array}{c}0.63 \\
(1.06)^{\mathrm{a}}\end{array}$ & $\begin{array}{c}0.75 \\
(1.12)^{\mathrm{a}}\end{array}$ \\
\hline $\mathbf{T}_{14}$ & Biodigester & $\begin{array}{l}1: 3 \\
\text { (Biodigester } \\
\text { :water) }\end{array}$ & $\begin{array}{c}0.55 \\
(1.02)\end{array}$ & $\begin{array}{c}0.49 \\
(0.99)^{\mathrm{ab}}\end{array}$ & $\begin{array}{c}0.53 \\
(1.01)^{\mathrm{ab}}\end{array}$ & $\begin{array}{c}0.53 \\
(1.01)^{b}\end{array}$ \\
\hline & S.Em \pm & & & 0.033 & 0.033 & 0.035 \\
\hline & CD@5\% & & NS & 0.10 & 0.11 & 0.11 \\
\hline & $\mathrm{CV}(\%)$ & & & 6.05 & 6.16 & 6.40 \\
\hline
\end{tabular}


Table.4 Population of Chrysoperla zasrteowi sillemi in chemical sprayed plot in safflower

\begin{tabular}{|c|c|c|c|c|c|c|}
\hline \multirow[t]{2}{*}{$\begin{array}{l}\text { Tr. } \\
\text { No. }\end{array}$} & \multirow[t]{2}{*}{ Treatment } & & \multicolumn{4}{|c|}{$\begin{array}{c}\text { Mean larval population of } C . \text { zasrteowi } \\
\text { per plant }\end{array}$} \\
\hline & & Dosage & DBT & $3 \mathrm{DAT}$ & $10 \mathrm{DAT}$ & $15 \mathrm{DAT}$ \\
\hline $\mathbf{T}_{1}$ & Profenophos 50 EC & $2 \mathrm{ml} / 1$ & $\begin{array}{c}0.53 \\
(1.01)\end{array}$ & $\begin{array}{c}0.32 \\
(0.91)^{b}\end{array}$ & $\begin{array}{c}0.32 \\
(0.91)^{\mathrm{b}}\end{array}$ & $\begin{array}{c}0.30 \\
(0.89)^{\mathrm{b}}\end{array}$ \\
\hline $\mathbf{T}_{2}$ & Acephate $75 \mathrm{SP}$ & $1 \mathrm{gm} / 1$ & $\begin{array}{c}0.47 \\
(0.98)\end{array}$ & $\begin{array}{c}0.31 \\
(0.90)^{b}\end{array}$ & $\begin{array}{c}0.29 \\
(0.88)^{b}\end{array}$ & $\begin{array}{c}0.28 \\
(0.88)^{b}\end{array}$ \\
\hline $\mathbf{T}_{\mathbf{3}}$ & NSKE $5 \%$ & $5 \mathrm{ml} / 1$ & $\begin{array}{c}0.51 \\
(1.00)\end{array}$ & $\begin{array}{c}0.45 \\
(0.98)^{\mathrm{ab}}\end{array}$ & $\begin{array}{c}0.44 \\
(0.97)^{\mathrm{ab}}\end{array}$ & $\begin{array}{c}0.43 \\
(0.97)^{\mathrm{ab}}\end{array}$ \\
\hline $\mathbf{T}_{4}$ & Deltamethrin 10EC & $0.5 \mathrm{ml} / \mathrm{l}$ & $\begin{array}{c}0.58 \\
(1.04)\end{array}$ & $\begin{array}{c}0.35 \\
(0.92)^{b}\end{array}$ & $\begin{array}{c}0.34 \\
(0.92)^{b}\end{array}$ & $\begin{array}{c}0.34 \\
(0.92)^{b}\end{array}$ \\
\hline $\mathbf{T}_{5}$ & NPV $2.0 \%$ AS & $250 \mathrm{LE} / \mathrm{ha}$ & $\begin{array}{c}0.48 \\
(0.99)\end{array}$ & $\begin{array}{c}0.44 \\
(0.97)^{\mathrm{ab}}\end{array}$ & $\begin{array}{c}0.42 \\
(0.96)^{\mathrm{ab}}\end{array}$ & $\begin{array}{c}0.42 \\
(0.96)^{\mathrm{ab}}\end{array}$ \\
\hline $\mathbf{T}_{6}$ & Btk $0.5 \% \mathrm{WP}$ & $1 \mathrm{gm} / 1$ & $\begin{array}{c}0.56 \\
(0.93)\end{array}$ & $\begin{array}{c}0.45 \\
(0.97)^{\mathrm{ab}}\end{array}$ & $\begin{array}{c}0.44 \\
(0.97)^{\mathrm{ab}}\end{array}$ & $\begin{array}{c}0.43 \\
(0.97)^{\mathrm{ab}}\end{array}$ \\
\hline $\mathbf{T}_{7}$ & Novaluron 10 EC & $0.75 \mathrm{ml} / 1$ & $\begin{array}{c}0.49 \\
(0.99)\end{array}$ & $\begin{array}{c}0.31 \\
(0.90)^{b}\end{array}$ & $\begin{array}{c}0.29 \\
(0.88)^{\mathrm{b}}\end{array}$ & $\begin{array}{c}0.28 \\
(0.88)^{b}\end{array}$ \\
\hline $\mathbf{T}_{8}$ & Rynaxypyr 20 SC & $0.15 \mathrm{ml} / 1$ & $\begin{array}{c}0.47 \\
(0.98)\end{array}$ & $\begin{array}{c}0.30 \\
(0.89)^{b}\end{array}$ & $\begin{array}{c}0.28 \\
(0.88)^{b}\end{array}$ & $\begin{array}{c}0.27 \\
(0.88)^{b}\end{array}$ \\
\hline $\mathbf{T}_{9}$ & Spinosad 45 SC & $0.1 \mathrm{ml} / \mathrm{l}$ & $\begin{array}{c}0.52 \\
(1.01)\end{array}$ & $\begin{array}{c}0.32 \\
(0.91)^{b}\end{array}$ & $\begin{array}{c}0.30 \\
(0.89)^{b}\end{array}$ & $\begin{array}{c}0.28 \\
(0.88)^{b}\end{array}$ \\
\hline $\mathbf{T}_{10}$ & Indoxacarb 14.5 SC & $0.3 \mathrm{ml} / 1$ & $\begin{array}{c}0.57 \\
(1.03)\end{array}$ & $\begin{array}{c}0.34 \\
(0.92)^{b}\end{array}$ & $\begin{array}{c}0.33 \\
(0.91)^{b}\end{array}$ & $\begin{array}{c}0.33 \\
(0.91)^{b}\end{array}$ \\
\hline $\mathbf{T}_{11}$ & $\begin{array}{l}\text { Emamectin benzoate } \\
5 \mathrm{SG}\end{array}$ & $0.2 \mathrm{~g} / \mathrm{l}$ & $\begin{array}{c}0.51 \\
(1.00)\end{array}$ & $\begin{array}{c}0.32 \\
(0.91)^{b}\end{array}$ & $\begin{array}{c}0.29 \\
(0.88)^{b}\end{array}$ & $\begin{array}{c}0.30 \\
(0.89)^{\mathrm{b}}\end{array}$ \\
\hline $\mathbf{T}_{12}$ & Flubendiamide $480 \mathrm{SC}$ & $0.075 \mathrm{ml} / 1$ & $\begin{array}{c}0.55 \\
(1.02)\end{array}$ & $\begin{array}{c}0.33 \\
(0.91)^{b}\end{array}$ & $\begin{array}{c}0.31 \\
(0.90)^{b}\end{array}$ & $\begin{array}{c}0.31 \\
(0.90)^{b}\end{array}$ \\
\hline $\mathbf{T}_{13}$ & Control & --- & $\begin{array}{c}0.58 \\
(1.04)\end{array}$ & $\begin{array}{c}0.69 \\
(1.09)^{\mathrm{a}}\end{array}$ & $\begin{array}{c}0.74 \\
(1.11)^{\mathrm{a}}\end{array}$ & $\begin{array}{c}0.82 \\
(1.15)^{\mathrm{a}}\end{array}$ \\
\hline $\mathbf{T}_{14}$ & Biodigester & $\begin{array}{l}1: 3 \\
\text { (Biodigester } \\
\text { :water) }\end{array}$ & $\begin{array}{c}0.52 \\
(1.01)\end{array}$ & $\begin{array}{c}0.49 \\
(0.99)^{\mathrm{ab}}\end{array}$ & $\begin{array}{c}0.48 \\
(0.99)^{\mathrm{ab}}\end{array}$ & $\begin{array}{c}0.48 \\
(0.99)^{\mathrm{ab}}\end{array}$ \\
\hline & S.Em \pm & & & 0.035 & 0.035 & 0.033 \\
\hline & CD@5\% & & NS & 0.11 & 0.10 & 0.10 \\
\hline & $\mathrm{CV}(\%)$ & & & 6.41 & 6.54 & 6.20 \\
\hline
\end{tabular}


Consequently the net profit derived out of different treatments was the highest Rs. 22724 to the lowest Rs. 13580 in rynaxypyr and biodigester, respectively. The highest B:C ratio was registered in rynaxypyr 20 SC @ $0.15 \mathrm{ml} / 1$ (1:1.98) followed by $1: 1.96$ in emamectinbenzoate 5\% SG @ $0.20 \mathrm{gm} / 1$ and $1: 1.93$ in spinosad $45 \mathrm{SC} @ 0.10 \mathrm{ml} / \mathrm{l}$. In the control plot, the lowest $\mathrm{B}: \mathrm{C}$ ratio of 1:0.90 was recorded.

Deshmukh et al., (2010) who reported the highest grain yield of $1850 \mathrm{~kg} / \mathrm{ha}$ in flubendiamide $0.007 \%$ and $1665 \mathrm{~kg}$ /ha in emamectin benzoate @ $0.00015 \%$ treated chickpea plots and gave the highest benefit cost ratio of 6.10 and 4.24, respectively. The rynaxypyr $20 \mathrm{SC}$ recorded the high yield of pigeonpea as reported by Satpute and Barkhade (2012). Patil et al., (2007) reported that the application of emamectin benzoate recorded higher cost benefit cost ratio of 2.27 in chickpea.

The data on the natural enemy population revealed that good numbers of coccinellids and $C$. zastrowi Sillemi population in all organic product treatments. The highest population of natural enemies was noticed in biodigester treatment followed by NSKE 5\%, NPV @ 250 LE/ha, Btk @ 1ml/l (Table 3). Even though slightly higher natural enemy population was observed in untreated control, there was no significant difference when compared with any of the organic treatments (Table 4).

The present findings are in line with Hanamantharaya et al., (2008) who reported that number of natural enemies (coccinelid and C.carnea) population in absolute control was 3.0 per plant which was superior over in profenophos treated safflower plot (0.90/plant).

From the above conducted experiment, it can be concluded that rynaxypyr, emamectinbenzoate, novaluron and flubendiamide were very effective against $H$. armigera on safflower with reduced damage and enhanced seed yields. The predatory population (Coccinellids and C.zasrteowi sillemi) in organic treated plots was statistically on par with control plot.

\section{References}

Anonymous, 1995, FAO, Production year book, 49: p115.

Deshmukh, S. G., Sureja, B. V., Jethva, D. M. and Chatter V. P., 2010, Field efficacy of different insecticides against Helicoverpa armigera (Hubner) infesting chickpea. Legume Res., 33 (4): 269-273.

Hanumantharaya L., Venkateshalu, Kubasad V.S and Raju S.G., 2008, Pest Status of safflower, Carthamus tinctorius L. in Northern Parts of Karnataka. VII International Safflower Conference, Waga Waga, Australia

Jadhav, J. D., Kadam, J. R., Khadtare, S.V. and Shinde, V. A., 2012, Need of ITK based rainfall analysis for safflower sowing under climate change situation in Maharashtra. J. Oilseeds Res., 29(1): 58-64.

Karve, A. D., Murugkar, V. K., and Quadri, S. M., 1978. Assessment of yield loss (reduction) due to aphid infestation. Report on resistance of safflower (Carthamus tinctorius L.) to insect and disease. Nimbkar Agric. Res. Inst., Phaltan, pp. 58-60.

Patil R. H and Halolli S. P., (2005), Proceedings of the VIth International Safflower Conference, Istanbul-Turkey, 6-10 June, 2005. Safflower: A unique crop for oil spices and health consequently, a better lifr for you 2005 pp. 229-235.

Patil, S. K., Ingle, M. B. and Jamadagni, B. M., 2007, Bio-efficacy and economics of insecticides for the management of 
Helicoverpa armigera (Hubner) in chickpea. Ann. Pl. Protec. Sci., 15 (2): 307-311.

Rajesh, C. L., Bheemanna, M., Ranjith Kumar, L., Arunkumar, H. and Vijaykumar, G., 2010, Baseline susceptibility studies of rynaxypyr 20 SC against okra borer, Helicoverpa armiger (Hubner). Resist. pest manage. Newslett., 20:19-21.
Satpute, N. S. and Barkhade, U. P., 2012, Evaluation of rynaxypyr $20 \mathrm{SC}$ against pigeonpea pod borer complex. J. Food Legumes, 25(2): 162-163.

Sekhar, P. R. and Rai, P. S., 1989, Studies on the biology and occurrence of natural enemies of safflower caterpillar, Prospalta conducta Walker (Lepidoptera: Noctuidae) in Karnataka (India). J. Res., 17: 19-22.

\section{How to cite this article:}

Biradarpatil, K.A. and Jagginavar, S.B. 2018. Bio efficacy of Newer Insecticide Molecules against Safflower Capsule Borer, Helicoverpa armigera (Hubner).

Int.J.Curr.Microbiol.App.Sci. 7(07): 316-323. doi: https://doi.org/10.20546/ijcmas.2018.707.038 